\title{
Fractal Strategy: Responding to COVID-19 Effectively on Multiple Timescales
}

As the COVID-19 outbreak spread across the globe, organizations were initially focused on reacting to the immediate issues posed by the epidemic. However, as the crisis unfolded, several other challenges rapidly emerged: preparing for a potential recession in the near term; anticipating an eventual rebound in demand (initially when social distancing restrictions are relaxed, and later when consumer and business confidence returns); and ultimately reimagining operations, products, and business models for the post-crisis world.

Crucially, even in the early stages of the outbreak, leaders could wait to take on these challenges sequentially - they already had to begin considering and acting on the longer-term implications of the crisis, due to the lead times required to build and execute, and the speed and unpredictability with which events can unfold. During critical periods in history, pivotal changes have often been concentrated in a very short period of time, and the "new normal" can arrive very rapidly. We also have the agency to shape history provided we act preemptively. In the case of COVID-19, as consumers adjusted to life under social distancing restrictions, they formed new habits that may persist long after the outbreak, and businesses' actions during the crisis shaped the formation of those behaviors.

In other words, leaders and organizations need to consider all levels of strategic response - reaction, recession, rebound, and re-imagination - simultaneously. Yet our benchmarking shows that many companies delayed responding to the longer-term implications to focus first on more immediate issues.

This is an example of a more general challenge that business leaders increasingly face: the need to think and operate on multiple timescales simultaneously. Companies need to manage longer timescales to avoid potential disruption from social, technological, or economic shifts. (See Chapter 11, "The Challenge of Slow.") And they need to manage shorter timescales to perform well in the current business to maintain viability and fund long term opportunities, especially as competitive advantage becomes less persistent. ${ }^{1}$

1 https://sloanreview.mit.edu/article/fighting-the-gravity-of-average-performance/. 
This challenge has increased in a world where business-relevant timescales have been stretched in both directions - from the speed of algorithms (operating on milliseconds) to the increased importance of planetary and social timescales (operating over decades). And critical timescales are now converging: issues that could previously be considered far off in the future, such as climate change, have reached a point where they directly affect and require action from business today. As the response to COVID-19 shows, companies' traditional methods for managing multiple timescales are often not up to this challenge.

\section{Companies Primarily Focused on Near-Term Responses}

To better understand how businesses have structured their response to the COVID-19 crisis, we conducted a survey of more than 300 companies across 55 countries and 24 industries in March and April of 2020. Not surprisingly, we found that nearly all companies had reacted to the immediate threats: About $85 \%$ of companies had taken multiple measures to protect the health and safety of employees and ensure business continuity.

Roughly $60 \%$ of companies had taken measures to plan for and navigate a likely recession, such as putting cost control measures in place and revising capital investment plans. In contrast, only about $40 \%$ of companies had taken measures to prepare for a potential rebound of demand, and very few had begun reimagining the business for a post-COVID-19 world.

And while the distinct challenges of reaction, recession, rebound, and reimagination will likely affect all businesses, only about $10 \%$ of survey respondents had started planning on all four pillars simultaneously (Figure 13.1).

\section{Traditional Mechanisms for Managing Multiple Time Scales Fall Short}

Companies do have a few traditional ways for balancing multiple timescales. However, the extreme challenges posed by COVID-19 have exposed their limitations.

One method is applying a discount rate to expected cashflows and computing a net present value for various options in order to make choices across different 


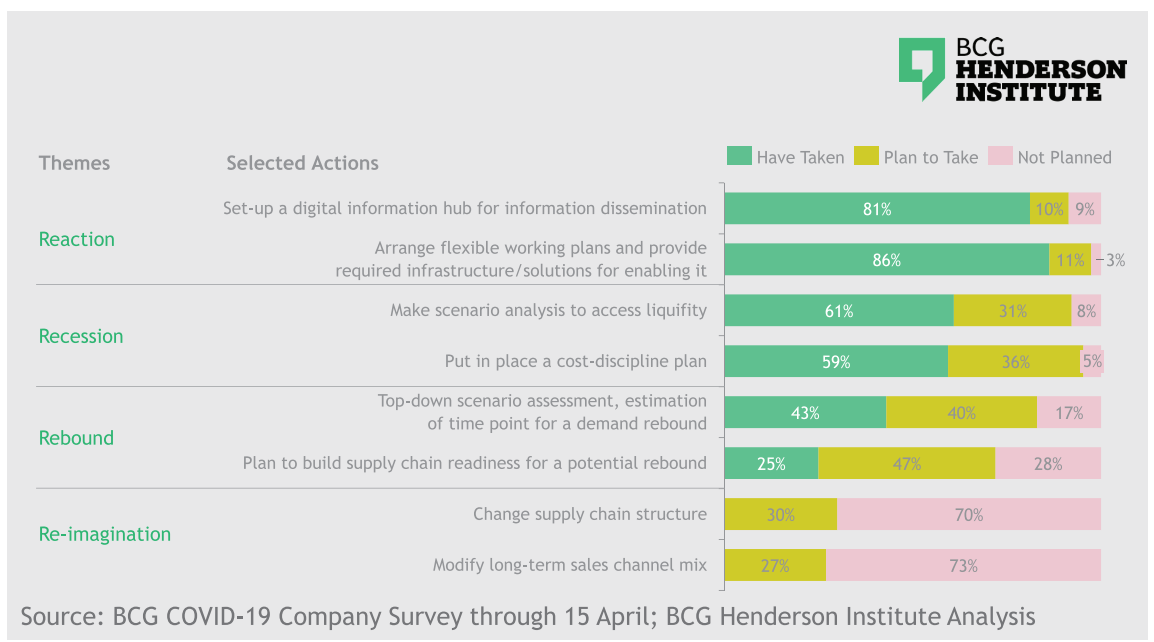

Figure 13.1: How businesses have structured their response to the COVID-19 crisis.

timescales. But many opportunities cannot be easily quantified in this way. For instance, potential long-term shifts in customer behaviors that result from COVID-19 can be hard to calibrate and there are multiple plausible scenarios. Human decision-makers are also known to apply hyperbolic discounting (applying a different discount rate at different times), which can distort time trade-offs. This can become more pronounced in the heat of a crisis, when the focus shifts to the short term. Finally, discounting is complicated by path dependence, in which actions taken today will shape which opportunities are available tomorrow.

Organizational hierarchy is another implicit mechanism for managing multiple timescales: employees at operational levels focus on day-to-day issues while senior leaders focus on longer-term strategic decisions. However, if a short-term issue arises that requires immediate structural action, hierarchical organizations can lack reliable override mechanisms to reallocate issues between levels. And when senior leaders are spurred to act against immediate threats, the balance can then shift away from longer-term opportunities, as demonstrated by many organizations' responses to COVID-19. Furthermore, there are time lags in transmitting and translating information up and down the hierarchy.

Some organizations have mixed planning cycles on different, fixed timescales, such as a detailed annual operating plan and a three- or five-year strategic plan. However, pre-determined timescales may not be appropriate for each specific situation. For instance, construction projects typically operate on extended planning cycles, but Chinese construction firm CSCEC adopted a 
24-hour cadence to construct two hospitals for coronavirus patients in ten days. Strategy processes need to be modulated according to the drumbeat set by the environment, rather than be fixed according to internal precedent.

Finally, companies often have separate units focused on different timescales. For example, sales, development, and research usually have very different time horizons. However, the timescales of business are becoming more continuous, expanded and intertwined, and a fixed number of pre-determined timeframes may not reflect important shifts, such as the emergence of new, algorithmic timescales. Moreover, the "long term" and the "short term" can converge as phenomena speed up. For example, new medical products are usually developed on multi-year cycles, but the COVID-19 crisis demanded greatly accelerated timelines. Finally, the relevant timeframe may not be predictable (as in the case of potential new waves of infections) and thus cannot be boxed into any fixed timescale.

\section{Strategy on Multiple Timescales}

A fundamental challenge in realizing a multi-timescale strategy is that different timescales call for different strategic approaches. We have previously identified five distinct approaches to strategy and execution which reflect the predictability, malleability, and harshness of the business environment to which they are applied (see Chapter 8, "Your Strategy Process Needs a Strategy"):

1. Classical strategy: For markets that are predictable, analyze the drivers, plan your actions, and execute against stable plans.

2. Adaptive strategy: For markets that are unpredictable, vary your bets, select the most promising ones, and scale them up quickly to capitalize on shifting opportunities.

3. Visionary strategy: For markets that can be predicted and shaped, envision the future, build a business to pursue the defined vision, and persist in pursuing that goal.

4. Shaping strategy: For markets that can be shaped but not predicted, engage a broad ecosystem of partners, orchestrate their actions, and co-evolve together.

5. Renewal strategy: For harsh markets and situations (such as a crisis or recession), react promptly and pragmatically to avoid danger, economize on resources, and later pivot to growth.

The COVID-19 crisis illustrates how issues on different timescales often require the application of different strategic approaches. For many large companies, day- 
to-day operations and demand have tended to be highly predictable, so a classical strategy based on planning and disciplined execution often dominates. However, during the COVID-19 crisis, markets became unpredictable even on short timescales, requiring an adaptive approach, focused on rapid learning and real-time adjustment. For example, as the outbreak began in Italy, energy infrastructure operator SNAM recognized the risk to its dispatching center, which manages gas distribution. ${ }^{2}$ It adapted rapidly, purchasing residential facilities, testing all dispatching center staff, and identifying a group of healthy employees to work and live on the premises. This response ensured that dispatching center staff were protected from the outbreak even as it intensified in surrounding area, and operational viability was maintained.

Over timescales of a few months, the effects of government-imposed lockdowns and a recession threatened many businesses' survival, forcing them to operate a renewal strategy. And while there is high uncertainty about the postcrisis future, consumer preferences will likely be malleable as they adjust to the forced changes in their lifestyles, calling for a visionary or shaping strategy.

The crisis also illustrates how business relevant timescales can rapidly change or converge. For example, pymetrics, a neuroscience/AI-based hiring and talent deployment start-up, initially developed a product roadmap for the year that prioritized its hiring support offering. However, many companies reduced hiring plans as the outbreak unfolded and instead focused on redeploying their existing workforce. As a result, pymetrics shifted to focus on redeployment offerings, which had previously been considered a longer-term priority. ${ }^{3}$ The company worked together with partners to build and orchestrate a platform for matching furloughed workers to job opportunities.

As new timescales emerge and existing timescales converge, businesses need to cultivate temporal ambidexterity - the ability to apply different strategic approaches to different problems on different timescales, and to modulate the approach according to changing circumstances.

2 https://bcghendersoninstitute.com/lessons-from-the-covid-crisis-marco-alver\%C3\%A0-ceo-ofsnam-3a5287063353.

3 https://bcghendersoninstitute.com/leading-through-the-covid-crisis-frida-polli-ceo-of-pymetrics -5c361a742550. 


\section{Realizing Fractal Strategy}

\section{Understand the Required Clock Speed and Strategic Approach of Each Business}

Within any company, each business line or geographic unit may have different relevant timescales and require correspondingly different approaches to strategy. Hence, leaders need to identify and apply the right strategic approaches in the right place at the right time. For example, consumer packaged goods companies are generally used to operating in a classical environment with predictable demand. But some business lines, such as health and safety products, faced temporary spikes in short-term demand, requiring an adaptive approach. Other business units may have been more stable in the short run but were exposed to long-term changes in distribution models as consumer activity shifted online, perhaps requiring a shaping strategy to collaborate on new channels and partnerships.

\section{Master the Capabilities Underpinning Different Approaches to Strategy}

Leaders in established companies are often used to working in classical (stable, predictable) environments, but a classical approach is not always optimal. In order to address challenges on all timescales, a company must be able to apply the entire palette of strategic approaches. Traditional top-down strategy is not feasible on algorithmic timescales; companies must instead take an adaptive approach, integrating AI, data platforms, and decision systems in autonomous learning loops. For example, global food giant Danone S.A. dealt with supply chain uncertainties by feeding AI tools with new data on COVID-19 spread patterns to help distribute materials and keep their factories running. And for slow-moving issues, classical strategy will miss opportunities to envision and shape the market for advantage, such as harnessing the power of organizational imagination.

\section{Master the Ability to Act on Multiple Timescales Simultaneously}

Balancing short-term exploitation and long-term exploration is a perennial business challenge, made more critical by the emergence and convergence of new timescales. Businesses face many short-term challenges that naturally demand leaders' attention. However, our research shows companies that act preemptively 
in the face of disruptions outperform those who wait longer, reinforcing the need to attend to longer-term issues early. ${ }^{4}$ For instance, in response to the COVID-19 crisis, LEGO set up a small team to think about the post-crisis future even while most of management was still focused on steering through the immediate crisis. ${ }^{5}$

As the business environment becomes more complex and diverse, traditional approaches to strategy will be increasingly limiting. Businesses can use crises like COVID-19 as an opportunity to revisit and strengthen their approaches to strategy. By being able to vary the clock speed of each part of the business and think on multiple timescales simultaneously, leaders can improve their odds of succeeding in this crisis and the next one.

\section{Coda: Inspiration from Biology}

Companies need to operate on multiple timescales to respond to the biological threat posed by the novel coronavirus. But they can also turn to biology for inspiration. Biological systems have evolved to survive both short- and long-run challenges, and their behaviors illustrate some key principles of a multi-timescale strategy (Figure 13.2).

\section{Foraging animals optimize for multiple timescales}
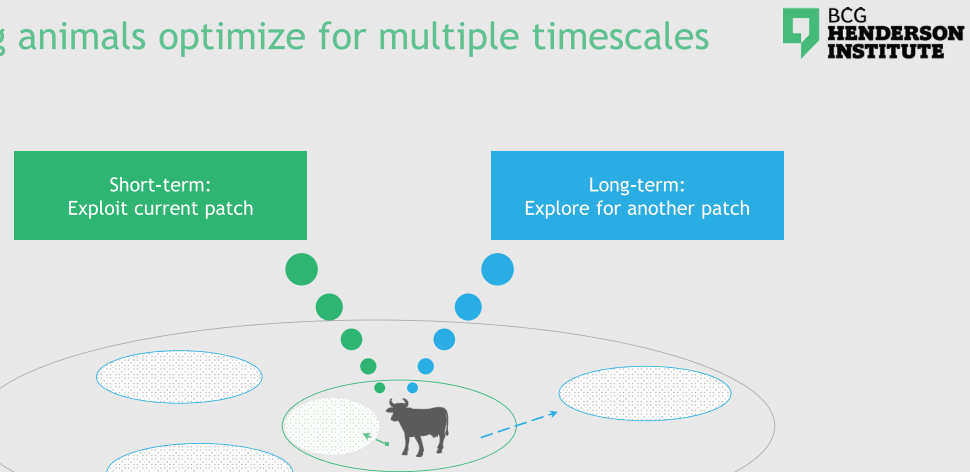

Source: "Preemptive Innovation: Leaping Before the Platform Burns" https://www.bcg.com/ publications/2018/leaping-before-platform-burns-increasing-necessity-preemptive-innovation

Figure 13.2: Biological systems illustrate some key principles of a multi-timescale strategy.

4 https://www.bcg.com/publications/2018/preemptive-transformation-fix-it-before-it-breaks. 5 https://bcghendersoninstitute.com/lessons-from-the-covid-crisis-j\%C3\%B8rgen-vig-knudstorpchairman-of-lego-brand-group-ab10ea135c60. 
Optimizing for multiple timescales: Animals that forage in patchy environments face a trade-off between feeding from their current patch (which provides short-term benefit but eventually exhausts that patch) and searching for a new patch (which incurs immediate costs but offers greater long term security). Many animals therefore begin searching for a new patch at the optimal point to balance short- and long-term benefits - an example of what is known as the Marginal Value Theorem. ${ }^{6}$ When is the right time for your business to start looking for a new patch?

Matching response mechanisms to timescales: Organisms respond to a change in the environment by making adjustments that cascade from short-term to longterm with decreasing reversibility, using different mechanisms (Figure 13.3). ${ }^{7}$ For example, if the environment becomes colder, animals might immediately shiver, which is fast and easily reversible. But if cooling persists, they will make more substantial adjustments, such as sheltering, moving to warmer places, or increasing fat storage, which are moderately reversible. If changes persist, they may eventually evolve genetically to become better adapted to cold environments. What are the cascading adaptation mechanisms for your business?

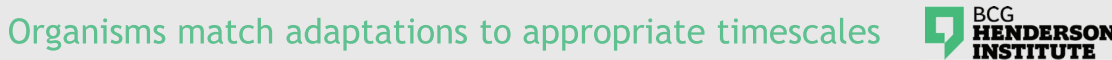

Cascading adaptations to cooling environment

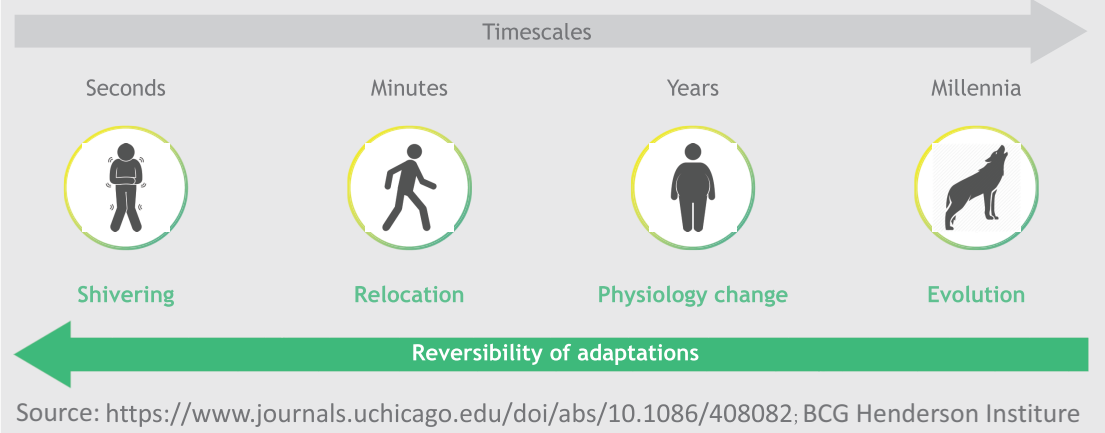

Figure 13.3: Organisms respond to a change in the environment by making adjustments that cascade from short-term to long-term with decreasing reversibility, using different mechanisms.

6 https://www.sciencedaily.com/releases/2011/06/110606152210.htm.

7 https://pubmed.ncbi.nlm.nih.gov/4411986/. 
Applying different types of strategies for different environments: Reproductive strategies can be divided two categories: r-selection, which involves producing many highly diverse offspring with low selection pressure and parental investment; and K-selection, which involves fewer offspring with higher parental investment. ${ }^{8}$ Species such as bacteria and rodents that operate in disrupted or uncertain environments tend to use r-selection strategies to ensure many "shots on goal," whereas species such as elephants or humans that operate in more stable environments tend to use K-selection strategies (Figure 13.4). Which innovation or new business build strategy should your business adopt in the current environment?

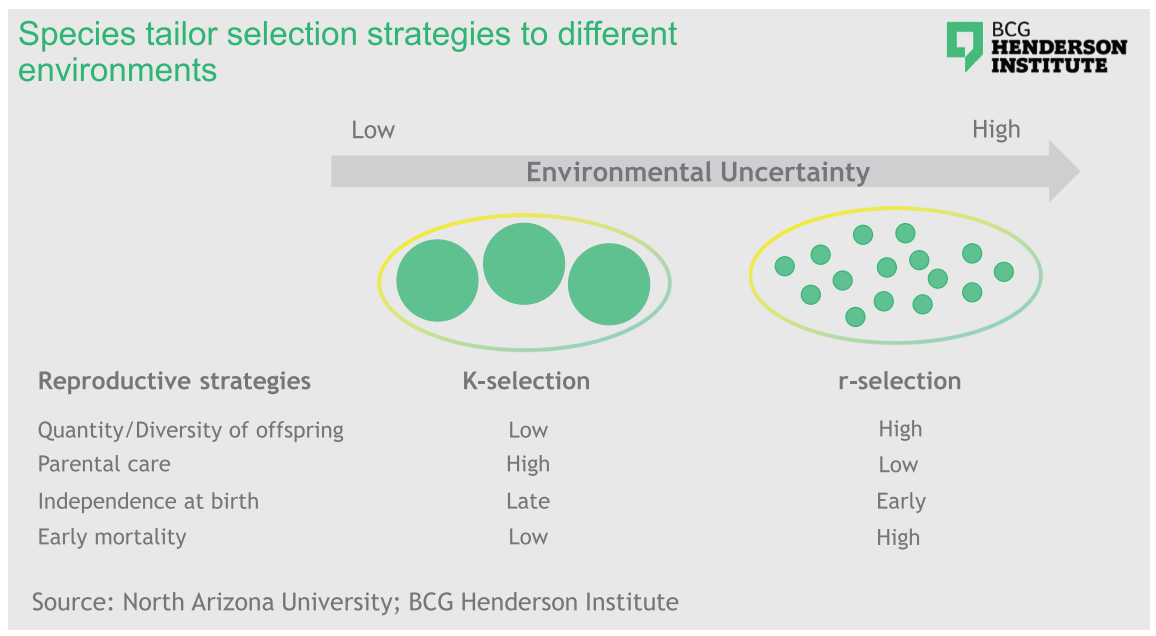

Figure 13.4: Reproductive strategies can be divided two categories: K-selection, which involves producing fewer offspring with higher parental investment; and r-selection, which involves more offspring with lower parental investment.

8 https://www.jstor.org/stable/2459020?seq=1\#metadata_info_tab_contents. 
